\title{
Treating alcohol withdrawal syndrome: Going beyond 'benzos'
}

Huma Baqir, MD, Sadiq Naveed, MD, and Saeed Ahmed, MD

A lcohol withdrawal syndrome (AWS) occurs when an individual who is alcohol-dependent suddenly stops or significantly reduces his/her alcohol intake. ${ }^{1}$ Symptoms of AWS, which can be fatal, include anxiety, restlessness, seizures, confusion, and delirium. ${ }^{1}$ Because benzodiazepines have been proven effective in improving symptoms of AWS, they are considered the first-line treatment, but they also carry the risk of abuse, psychomotor sedation, cognitive impairment, and interactions with alcohol. ${ }^{1}$

Non-benzodiazepine anticonvulsants (NBACs) such as valproic acid (VPA) and carbamazepine may offer benefit as alternatives or adjuncts to benzodiazepines. ${ }^{1}$ Many NBACs affect the functioning of glutamate and gamma-aminobutyric acid (GABA) neurotransmitters, ${ }^{2}$ which are particularly dysregulated in patients with AWS. Because NBACs help stabilize this imbalance, they may be useful for managing AWS and preventing relapse without the risks associated with benzodiazepines. ${ }^{2}$

\section{Valproic acid: A better choice than carbamazepine}

Compared with other NBACs, VPA and carbamazepine have been studied more extensively for treating patients with AWS, and their clinical effectiveness has been well documented. ${ }^{1}$ For mild-to-moderate AWS, VPA and carbamazepine may be as effective as benzodiazepines in reducing the severity of symptoms, and more potent for preventing withdrawal seizures. ${ }^{1}$

Increasing data suggests that compared with VPA, adjunctive treatment with carbamazepine for AWS may be more fre- quently associated with intolerable adverse effects such as ataxia, orthostatic hypotension, vertigo, nausea, and vomiting. ${ }^{1}$ The rapid onset of AWS requires rapid-acting pharmacotherapy. ${ }^{1}$ In attempting to rapidly achieve the desired plasma concentrations of carbamazepine, clinicians may risk inducing adverse effects. Compared with carbamazepine, VPA is associated with faster symptom resolution, a shorter duration of pharmacologic treatment, fewer transfers to the ICU, fewer withdrawal seizures, and a more favorable adverse effect profile. ${ }^{1}$ Likely due to its shorter half-life, VPA delivers its therapeutic effects without producing significant adverse effects. ${ }^{1}$

Early and aggressive treatment of AWS is needed to block kindling, ${ }^{3}$ which is characterized by the worsening of withdrawal symptoms each time an individual attempts to quit drinking alcohol. Compared with carbamazepine, VPA may be more helpful for blocking kindling. ${ }^{3}$

\section{More data is needed}

Due to ethical concerns, few studies have compared anticonvulsant medications with placebo for treating AWS. ${ }^{2}$ Most studies examining NBACs for AWS have either

continued on page 38

Every issue of CURRENT PsychIATRY has its 'Pearls'

\section{Yours could be found here.}

Read the 'Pearls' guidelines for manuscript submission at MDedge.com/ CurrentPsychiatry/page/pearls. Then, share with your peers a 'Pearl' of wisdom from your practice.
Dr. Baqir is a PGY-2 Resident Physician, Department of Psychiatry, University at Buffalo, New York. Dr. Naveed is Lead Child and Adolescent Psychiatrist, Inpatient Child and Adolescent Units, Institute of Living, Hartford, Connecticut. Dr. Ahmed is an Addiction Psychiatrist, and serves as Medical Director, West Ridge Center at Rutland Regional Medical Center, Rutland, Vermont. Disclosures

The authors report no financial relationships with any companies whose products are mentioned in this article, or with manufacturers of competing products.

doi: $10.12788 /$ cp.0066

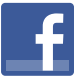

Discuss this article at www.facebook.com/ MDedgePsychiatry $\mathbf{0}$ 
Compared with carbamazepine, VPA may be more helpful for blocking kindling in AWS

\section{Alcohol withdrawal syndrome}

continued from page 27

used the anticonvulsant as an adjunct to a benzodiazepine to examine improvement in withdrawal symptoms, or compared the anticonvulsant with placebo or another intervention to assess the amount of a benzodiazepine required for safe detoxification. ${ }^{2,4}$ Additionally, most studies examining NBACs have been short, and few followed patients after the active medication period, which limits our knowledge of the long-term effectiveness of NBACs. ${ }^{2}$ Before NBACs can replace benzodiazepines for managing AWS, further evidence from clinical trials is needed to assess their efficiency as a stand-alone treatment.

\section{References}

1. Maldonado JR. Novel algorithms for the prophylaxis and management of alcohol withdrawal syndromesbeyond benzodiazepines. Crit Care Clin. 2017;33(3): 559-599.

2. Hammond CJ, Niciu MJ, Drew S, et al. Anticonvulsants for the treatment of alcohol withdrawal syndrome and alcohol use disorders. CNS Drugs. 2015;29(4):293-311.

3. Eyer F, Schreckenberg M, Hecht D, et al. Carbamazepine and valproate as adjuncts in the treatment of alcohol withdrawal syndrome: a retrospective cohort study. Alcohol Alcohol. 2011;46(2):177-184

4. Guirguis E, Richardson J, Kuhn T, et al. Treatment of severe alcohol withdrawal: a focus on adjunctive agents. J Pharm Technol. 2017;33(5):204-212 